\title{
THE FEASIBILITY OF DETECTING SUPERCOOLED LIQUID WITH A FORWARD-LOOKING RADIOMETER
}

\author{
Ian Stuart Adams* \\ NASA Goddard Space Flight Center \\ Mesoscale Atmospheric Processes Laboratory \\ Greenbelt, MD 20771
}

\author{
Justin Bobak \\ U.S. Naval Research Laboratory \\ Remote Sensing Division \\ Washington, D.C. 20375
}

\begin{abstract}
A three-dimensional radiative transfer model is utilized to determine the feasibility of a forward-viewing passive sensor for remotely detecting hazardous icing conditions. Wband ground-based radar simulations show no obvious ability to discriminate a cloud-top supercooled layer; however, the spectra for a forward-viewing passive sensor show a strong signal at two stand-off distances when compared with the clear sky spectrum. Such an instrument would be critical for manned and unmanned aircraft, particularly when size, weight, and power requirements restrict the installation of deicing equipment.
\end{abstract}

Index Terms - Radiometer, radar, clouds, supercooled liquid

\section{INTRODUCTION}

Supercooled liquid clouds constitute an appreciable climate feedback effect, and, as the climate warms, the presence of supercooled liquid is expected to increase. Such changes will alter the albedo of clouds; however, the partitioning of liquid and ice in mixed-phase clouds is poorly represented in global climate models. Beyond climate applications, supercooled liquid presents a hazard to aviation through airframe icing. To address both of these areas, improvements in the ability to detect and measure supercooled layers in mixedphase clouds are necessary, and simulation tools are required to interpret remote sensing observations of clouds. Radiative transfer modeling demonstrates a strong radiometric response to supercooled liquid layers that are not visible to groundbased $\mathrm{W}$-band radar.

\section{RADIATIVE TRANSFER MODEL DESCRIPTION}

To perform radiative transfer simulations, we used the Atmospheric Radiative Transfer Simulator (ARTS), version 2.3 [1]. For passive simulations, we simulated spectra from $30 \mathrm{GHz}$ to $200 \mathrm{GHz}$ using Monte Carlo integration, which accounts

*I.S. Adams was previously at the U.S. Naval Research Laboratory for scattering effects [2]. To simulate dual-polarization radar with finite antenna beams, we extended ARTS to calculate radar reflectivity using Monte Carlo sampling of a Gaussian antenna pattern to produce a finite beam response.

Hydrometeor scattering properties for ice particles are calculated using the T-Matrix method for fixed orientation [3] with a temperature-dependent complex permittivity for ice [4]. While T-Matrix calculations are not as representative as the higher-fidelity discrete dipole approximation (DDA) for frozen hydrometeors with irregular shape, a comprehensive database of DDA-computed particles is not available for horizontally-aligned snow particles. Although the size of water droplets are outside of the scattering regime for this study, spherical supercooled cloud liquid droplets are modeled as a scattering species using the T-Matrix for random orientations [5] with complex permittivity prescribed by Turner et al. [6] to produce the phase matrices necessary for radar reflectivity calculations. Gas absorption is calculated using the complete Rosenkranz absorption model [7, 8, 9], with improvements to oxygen absorption [10].

\section{CLOUD MODEL DESCRIPTION}

The cloud profile model is a simplistic expression of a mixedphase cloud observations [11]. The profile of liquid, pristine ice, and aggregated snow is shown in Fig. 1. All hydrometeor species are represented by a gamma distribution with shape parameter $\mu=3$. The effective radii for liquid cloud, pristine ice, and aggregated snow are $10 \mu \mathrm{m}, 20 \mu \mathrm{m}$, and $100 \mu \mathrm{m}$ respectively. The size of supercooled liquid droplets suggest spherical geometries. Pristine ice particles are modeled as cylindrical plates with a four-to-one aspect ratio, and aggregates are oblate spheroids with a 1.33-to-one aspect ratio. The von Mises distribution, which is a good directional statistics approximation of a Gaussian distribution, is used to prescribe particle flutter, with a Gaussian-equivalent standard deviation of $10^{\circ}$. As shown in the cloud profile, the vertical extent of the cloud is $1.5 \mathrm{~km}$. The three-dimensional nature of the radiative transfer model allows finite horizontal dimensions. The cloud extends $500 \mathrm{~km}$ in the north-south direction to approximate 


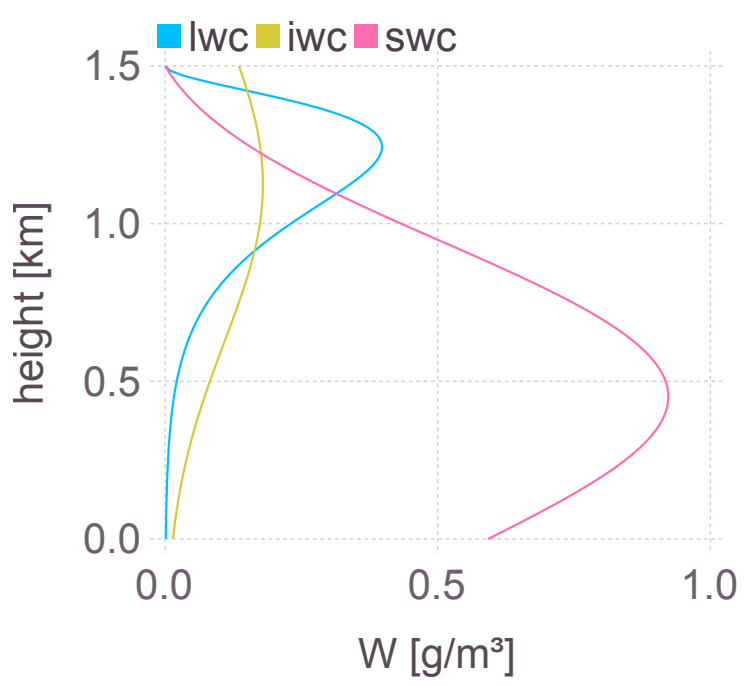

Fig. 1. Liquid, ice, and snow model profiles.

an infinitely long cloud, while the cloud is limited to $100 \mathrm{~km}$ in the east-west dimension.

\section{SIMULTATION RESULTS}

The simulation results show unique responses for both active and passive sensors, suggesting that synergistic observations can capture overarching features of subarctic mixed-phase clouds. Moreover, the three-dimensional domain geometry facilitates simulating the response of a forward-viewing radiometer designed to detect and quantify flight-path icing conditions for an aircraft approaching a mixed-phase cloud.

Radar reflectivities at $90 \mathrm{GHz}$ respond primarily to the larger aggregated snow as shown in Fig. 2; however, there is not an obvious response to the supercooled liquid or the pristine ice particles. By simulating a forward-viewing passive sensor at the level of the supercooled layer, we demonstrate a strong response at distances of $15 \mathrm{~km}$ and $30 \mathrm{~km}$ from the cloud edge, when compared to clear sky simulations (Fig. 3). The response to an ice-only cloud (not shown) deviated by only $1 \mathrm{~K}$ from the clear sky simulations.

\section{CONCLUSIONS}

The simulation results presented in this work demonstrate the feasibility of a forward-viewing passive sensor for avoiding hazardous flight conditions. Additionally, such measurements could be used for monitoring supercooled liquid layers that present an appreciable climate feedback mechanism. Of course, more comprehensive simulations over a wide range of conditions are necessary for determining the information

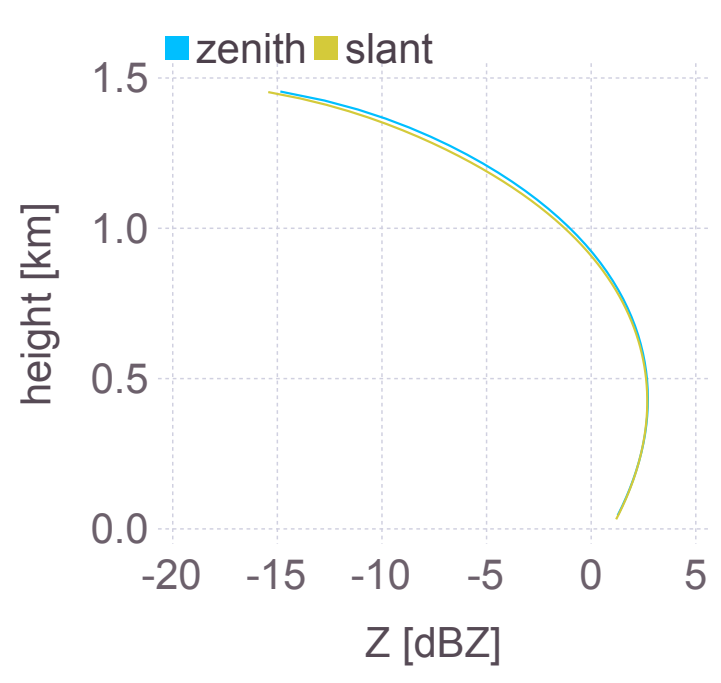

Fig. 2. Reflectivities for zenith and $45^{\circ}$ slant-path views.

content in the passive brightness temperature spectra for varying liquid and ice water contents and for precipitating supercooled conditions.

\section{REFERENCES}

[1] P. Eriksson, S.A. Buehler, C.P. Davis, C. Emde, and O. Lemke, "ARTS, the Atmospheric Radiative Transfer Simulator, version 2," J. Quant. Spectrosc. Rad. Trans., vol. 112, pp. 1551 - 1558, 2011.

[2] C Davis, C Emde, and R Harwood, "A 3D polarized reverse Monte Carlo radiative transfer model for $\mathrm{mm}$ and sub-mm passive remote sensing in cloudy atmospheres," IEEE Trans. Geosci. Remote Sens., vol. 43, pp. 10961101, May 2005.

[3] Michael I Mishchenko, "Calculation of the amplitude matrix for a nonspherical particle in fixed orientation," Appl. Opt., vol. 39, pp. 1026-1031, Feb 2000.

[4] C. Mätzler, "Dielectric properties of natural media: microwave dielectric properties of ice," in Thermal Microwave Radiation: Applications for Remote Sensing, C. Mätzler, Ed., pp. 455-463. The Institution of Engineering and Technology, 2006.

[5] Michael I Mishchenko and Larry D Travis, "Capabilities and limitations of a current FORTRAN implimentation of the T-matrix method for randomly oriented, rotationally symmetric scatterers," J. Quant. Spectrosc. Rad. Trans., vol. 60, pp. 309-324, Sep 1998. 


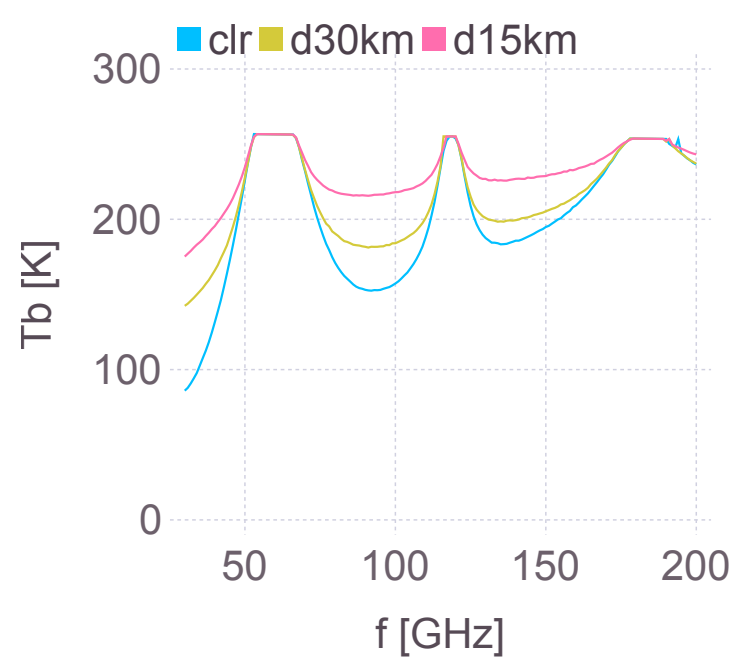

Fig. 3. Passive microwave $T_{b}$ spectra for a forward-viewing sensor.

[6] D.D. Turner, S. Kneifel, and M. P. Cadeddu, "An improved liquid water absorption model at microwave frequencies for supercooled liquid water clouds," J. Atmos. Oceanic Technol., vol. 33, pp. 33-44, Jan 2016.

[7] P W Rosenkranz, "Absorption of microwaves by atmospheric gasses," in Atmospheric Remote Sensing by Microwave Radiometry, M A Janssen, Ed., pp. 37-90. Wiley, 1993.

[8] P W Rosenkranz, "Water vapor microwave conitnuum absorption: A comparison of measurements and models," Radio Sci., vol. 33, pp. 919-928, Apr 1998.

[9] P W Rosenkranz, "Correction to 'water vapor microwave conitnuum absorption: A comparison of measurements and models'," Radio Sci., vol. 34, pp. 1025, Apr 1999.

[10] M. Yu. Tretyakov, M.A. Koshelev, V.V. Dorovskikh, D.S. Makarov, and P.W. Rosenkranz, "60-ghz oxygen band: precise broadening and central frequencies of fine-structure lines, absolute absorption profile at atmospheric pressure, and revision of mixing coefficients," J. Molec. Spectrosc., vol. 231, pp. 1-14, May 2005.

[11] M. D. Shupe, J. S. Daniel, G. de Boer, E. W. Eloranta, P. Kollias, C. N. Long, E. P. Luke, D. D. Turner, and J. Verlinde, "A focus on mixed-phase clouds," Bull. Amer. Meteor. Soc., vol. 89, pp. 1549-1562, Oct 2008. 